\title{
Pyoderma gangraenosum der Kopfhaut bei Colitis ulcerosa
}

\author{
Pyoderma gangrenosum of the Scalp in a Patient with Ulcerative Colitis
}

Autoren

Institut

\section{A. Shab, N. Deris, A. Wagner, C. Bayerl}

Klinik für Dermatologie und Allergologie, Wilhelm-Fresenius-Klinik, Dr. Horst- Schmidt- Kliniken, Städtisches Klinikum Wiesbaden

\section{Bibliografie}

DOI http://dx.doi.org/

10.1055/s-0030-1256536

Akt Dermatol 2011; 37:

254-257 @ Georg Thieme

Verlag KG Stuttgart · New York

ISSN 0340-2541

Korrespondenzadresse

Dr. med. Arna Shab

Klinik für Dermatologie und Allergologie

Dr. Horst-Schmidt-Klinik

Wiesbaden

Aukammallee 39

65191 Wiesbaden

Arna.Shab@HSK-Wiesbaden.de

\section{Zusammenfassung \\ $\nabla$}

Wir berichten über einen 42-jährigen, männlichen Patienten mit pustulösen Hautveränderungen am Hinterkopf, die während eines stationären Aufenthaltes in der Gastroenterologie zur Abklärung rezidivierender blutiger Durchfälle erstmalig auftraten und sich rasch zu größenprogredienten, schmerzhaften Ulzerationen entwickel-

\section{Einleitung}

$\nabla$

PG ist eine seltene, chronische, primär sterile, neutrophilenreiche, ulzerierende Hautkrankheit. Typischerweise treten zunächst sterile Pusteln auf, die sich rasch zu schmerzhaften Ulzerationen unterschiedlicher Größe und Tiefe entwickeln. Charakteristisch ist der unterminierte bläulichviolette, häufig druckschmerzhafte Randwall, der meist von einem Erythem und Ödem umgeben ist $[1,2]$. Besonders häufig sind die unteren Extremitäten betroffen, jedoch können die Ulzerationen an jeder beliebigen Lokalisation auftreten. Typisch sind Entwicklung neuer Läsionen und die Ausbreitungstendenz schon bestehender Ulzerationen nach einem minimalen Trauma, das sogenannte Pathergie-Phänomen $[3,11]$. Das Durchschnittsalter der Erkrankten liegt bei 40 Jahren [8]. Seit seiner Erstbeschreibung im Jahr 1930 von Brunsting et al. [10] ist die Ätiopathogenese weiterhin ungeklärt. Ca. 50\% der Fälle von PG sind mit einer zugrunde liegenden systemischen Erkrankung assoziiert [1]. Die häufigsten assoziierten Erkrankungen sind entzündliche Darmerkrankungen wie Morbus Crohn oder Colitis ulcerosa, rheumatologische oder hämatologische Erkrankungen und Malignome [4].

Wichtig für die Behandlung ist die korrekte Diagnose und die Identifikation und Behandlung vergesellschafteter Erkrankungen, sowie die Wahl angemessener topischer und systemischer Therapiemaßnahmen. Eine Lokaltherapie kann für ten. Nach Ausschluss anderer ulzerativer Prozesse stellten wir die Diagnose eines Pyoderma gangraenosum (PG). Die durchgeführte Diagnostik ergab als zugrunde liegende Erkrankung eine Colitis ulcerosa (CU). Eine systemische Glukokortikoidtherapie, begleitet von einer Mesalazingabe speziell zur Behandlung der $\mathrm{CU}$, und eine spätere Azathioprinmedikation führten zur Abheilung des PG.

milde Verlaufsformen ausreichen, während für schwere Fälle eine systemische immunsuppressive Therapie erforderlich ist.

\section{Kasuistik}

$\nabla$

\section{Anamnese und Befund}

Im August 2008 stellte sich ein 42-jähriger Patient mit seit 4 Wochen bestehenden Ulzerationen am Capillitium vor. Es zeigten sich am Hinterkopf 3 haselnussgroße Knoten mit spontaner Pusentleerung. Am gesamten Capillitium zeigten sich disseminiert Pusteln und Krusten.

Der Patient berichtete, dass er zuvor stationär in der Gastroenterologie zur Abklärung wochenlang blutiger Durchfälle gewesen war. Bei Verdacht auf eine Colitis ulcerosa wurde eine Koloskopie durchgeführt. Unter der Diagnose einer bakteriellen Enteritis wurde im Verlauf in der Gastroenterologie eine intravenöse Antibiose durchgeführt. Hierunter zeigten sich die Durchfälle regredient. Gleichzeitig traten dann erstmals Pusteln am Hinterkopf auf und entwickelten sich zu schmerzhaften Ulzerationen am Capillitium. Aufgrund eines externen positiven mykologischen Befundes wurde der Patient mit Griseofulvin behandelt. Bei fehlender Besserung des Hautbefundes und weiterer Progredienz begab er sich zur stationären Therapie in unsere Klinik. 


\section{Therapie und Verlauf}

Am Aufnahmetag erfolgte die Inzision und Pusentleerung der Knoten am Hinterkopf $(\boldsymbol{O}$ Abb. 1$)$. Unter dem Verdacht einer bakteriellen Superinfektion wurde eine systemische Antibiose eingeleitet. Der Lokalbefund blieb jedoch weiterhin progredient. Anamnestisch war ambulant ein Pilzbefall festgestellt worden. Im Nativpräparat bestätigte sich dies in unserer Klinik nicht, jedoch konnte eine tiefe Mykose differenzialdiagnostisch noch nicht ausgeschlossen werden, da die Pilzkultur ausstand. Eine prophylaktische systemische Antimykotikagabe (Griseofulvin, später Itraconazol) erfolgte. Die Lokaltherapie erfolgte mit CiclopiroxCreme. Der Hautbefund verschlechterte sich jedoch weiterhin. Es kam zur nekrotischen Einschmelzung der Kopfhaut und der Patient entwickelte Fieber. Aus diesem Grund wurde der Patient in der Allgemein- und Neurochirurgie vorgestellt und eine multiple Abszessoffenlegung mit Drainage durchgeführt.

Im weiteren stationären Aufenthalt traten immer wieder intermittierend Fieberschübe und auch wieder im zunehmenden Ausmaß Durchfälle auf. Außerdem entwickelte er neue, nekrotisch abzedierende Herde am Rücken. Da die Pilzkultur nach 2 Wochen negativ war, wurde die antimykotische Therapie beendet. In einer ebenfalls durchgeführten Probebiopsie zeigte sich ein von Granulozyten, Lymphozyten und Histiozyten durchsetztes Bild ohne Myceliennachweis. So wurde nach Ausschluss von Differenzialdiagnosen und durch das klinische Bild die Diagnose eines PG gestellt. Nach Eingang des histologischen Befundes und Beurteilung der durchgeführten Diagnostik ( $\bullet$ Tab. 1) wurde eine Therapie mit $50 \mathrm{mg}$ Solu Decortin H i.v. eingeleitet, die im weiteren Verlauf auf $80 \mathrm{mg}$ gesteigert wurde. Außerdem wurde eine ReKoloskopie angesetzt. Unter der diesmal gestellten Diagnose CU konnte eine Therapie mit Mesalazin $4 \mathrm{~g} / \mathrm{d}$ eingeleitet werden.

Die Lokaltherapie am Kopf erfolgte mit nekrolytischen Externa und Wunddressings. Zusätzlich erfolgten im Rahmen des stationären Aufenthaltes weitere Nekroseabtragungen sowie Anlage einer Vakuumversiegelung an der Kopfhaut unter Antibiotikaprophylaxe.

Unter systemischen und topischen Therapiemaßnahmen sistierten die Durchfälle, die Wunden granulierten gut ( $\bullet$ Abb. 2), die Entzündungsparameter waren rückläufig und der Allgemeinzustand des Patienten besserte sich deutlich. Die Steroiddosis wurde allmählich auf $30 \mathrm{mg}$ reduziert und eine Azathioprin-Therapie (100 mg $2 \times 1 / d$ ) ergänzend eingeleitet. Die Mesalazin-Dosis wurde auf $3 \mathrm{~g} / \mathrm{d}$ erniedrigt. Der Hautbefund besserte sich so weit, dass wir unseren Patienten nach 6 Wochen in die ambulante Betreuung entließen.

Ein halbes Jahr später stellen sich die Wunden reizlos dar $(\circlearrowleft$ Abb. 3). Decortin wurde ausgeschlichen und die AzathioprinTherapie fortgeführt (zurzeit bei $150 \mathrm{mg} / \mathrm{d}$ ). Darunter traten keine weiteren Rezidive auf.

\section{Diskussion}

\section{$\nabla$}

Das PG hat vier unterschiedliche klinische und histologische Varianten (ulzerös, pustulös, bullös und vegetierend), wobei das spezifische klinische Erscheinungsbild einen Schlüssel zu der zugrunde liegenden Erkrankung liefert [4,8]. Das ulzeröse PG ist die am häufigsten vorkommende Variante [12] und ist zu > 70\% mit systemischen Erkrankungen assoziiert. Entzündliche Darmerkrankungen findet man in $>30 \%$ der Fälle als zugrunde liegende Erkrankung [4]. Auch bei der pustulösen und der bullösen Form liegen in $>70 \%$ assoziierte Erkrankungen vor, wobei die pustu-

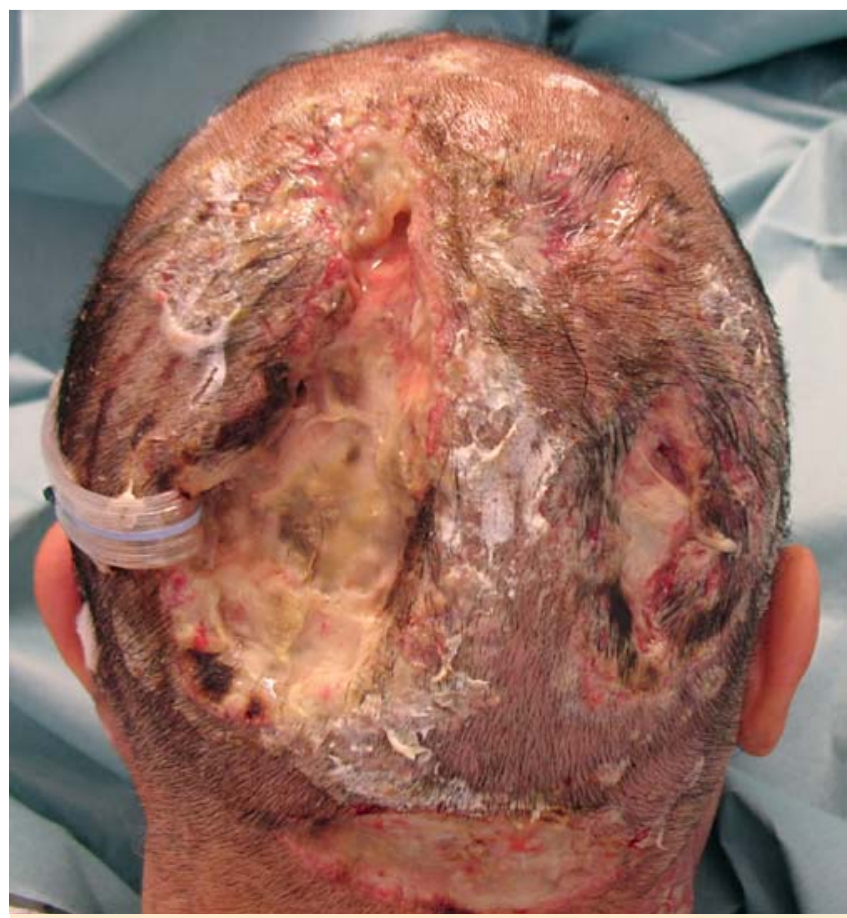

Abb. 1 Ulzeröses PG am Capillitium nach Inzision, radikaler Wundreinigung und Drainageeinlage zu Beginn des stationären Aufenthalts.

Tab. 1 Tabellarische Auflistung der Diagnostik.

\begin{tabular}{|c|c|}
\hline Diagnostik & Befunde \\
\hline Labor & $\begin{array}{l}\text { CRP 5,4 mg/dl; BSG 65/91 mm n. W.; } \\
\text { Leukozyten 7,73/nl; Erythrozyten 3,69/pl; } \\
\text { Hb 10,6 g/dl; Hkt 32,8\%; Thrombozyten } \\
\text { 520/nl; Gesamt IgE } 472 \text { kU/l }\end{array}$ \\
\hline Rheumaserologie & ASL $998 \mathrm{IU} / \mathrm{ml}$; Anti-DNase B $\leq 79 \mathrm{IU} / \mathrm{ml}$ \\
\hline p-ANCA und c-ANCA & negativ \\
\hline Hämoccult & positiv \\
\hline $\begin{array}{l}\text { Serum-Eiweiß-Elektro- } \\
\text { phorese }\end{array}$ & $\begin{array}{l}\text { Gesamteiweiß 5,8 g/dl; Albumin } 36 \% \text {; } \\
\alpha_{1} \text {-Globuline } 8,1 \% ; \alpha_{2} \text {-Globuline } 15,6 \% \text {; } \\
\beta \text {-Globuline } 9,2 \% ; \gamma \text {-Globuline 31,1\% }\end{array}$ \\
\hline Immunstatus & $\begin{array}{l}\text { Leukozytose mit Neutrophilie, normale } \\
\text { Lymphozytenzahl, Anzahl der gesamten } \\
\text { T-Zellen (CD3) geringgradig erniedrigt, } \\
\text { Anzahl der NK-Zellen (CD15/56) geringfügig } \\
\text { erhöht, Zahl der T-Helferzellen (CD4) sowie } \\
\text { der T-Suppressorzellen (CD8) unauffällig }\end{array}$ \\
\hline $\begin{array}{l}\text { Immunphäno- } \\
\text { typisierung }\end{array}$ & $\begin{array}{l}\text { stark reaktive Knochenmarksveränderungen } \\
\text { mit gesteigerter Granulopoese }\end{array}$ \\
\hline Durchflusszytometrie & $\begin{array}{l}\text { kein Anhalt für monoklonale B-Zellpopula- } \\
\text { tion, überwiegend Granulozyten }\end{array}$ \\
\hline $\begin{array}{l}\text { Blutkultur, mikrobio- } \\
\text { logische Abstriche, } \\
\text { mykologischer Befund }\end{array}$ & steril \\
\hline Re-Koloskopie & aktive, langjährig bestehende CU \\
\hline MRT/CT Schädel & $\begin{array}{l}\text { subkutane Nekroseareale bds occipital, mul- } \\
\text { tiple Lymphknoten nuchal und retroaurikulär }\end{array}$ \\
\hline Histologie & $\begin{array}{l}\text { schwergradige ulzerierende, phlegmonöse, } \\
\text { abszedierende, teilweise nekrotisierende } \\
\text { Entzündung }\end{array}$ \\
\hline
\end{tabular}

löse Variante häufig im Zusammenspiel mit entzündlichen Darmerkrankungen auftritt [4].

Die Diagnose einer der vier Formen des PG basiert auf dem typischen klinischen Erscheinungsbild, der Histopathologie, dem 


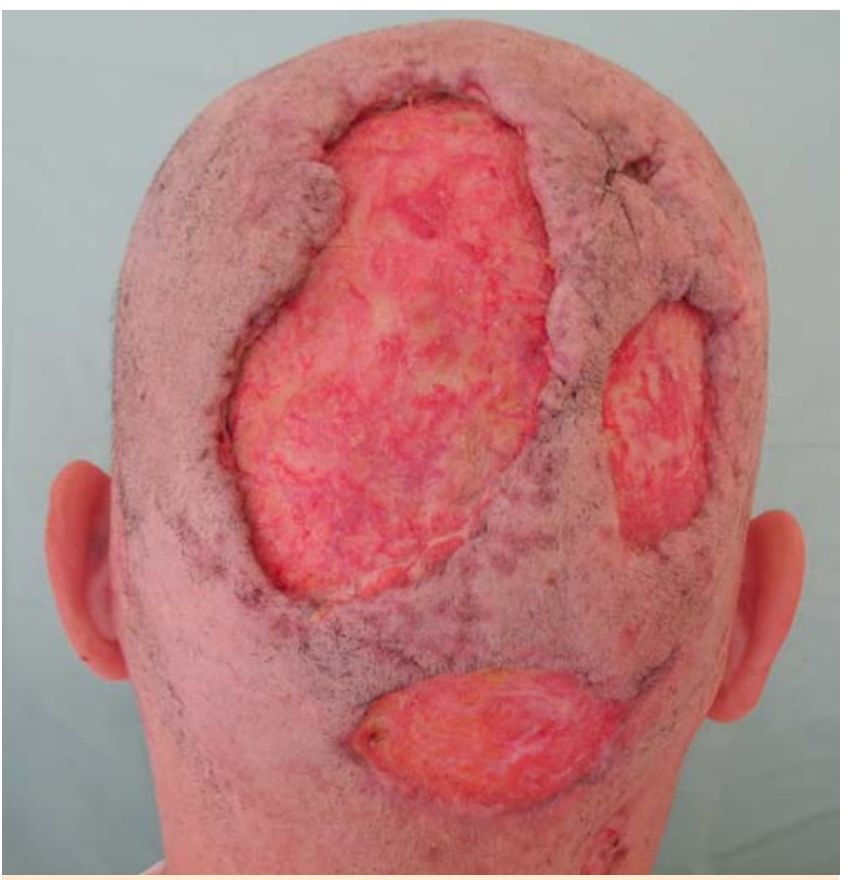

Abb. 2 Ulzeröses PG am Capillitium. Gute Granulation der Wunden 2 1⁄2 Wochen später nach mehrfacher Abszessoffenlegung, Drainage, Nekroseabtragung und Vakuumversiegelung der Kopfhaut sowie einer systemischen Therapie mit Solu Decortin H und Mesalazin.

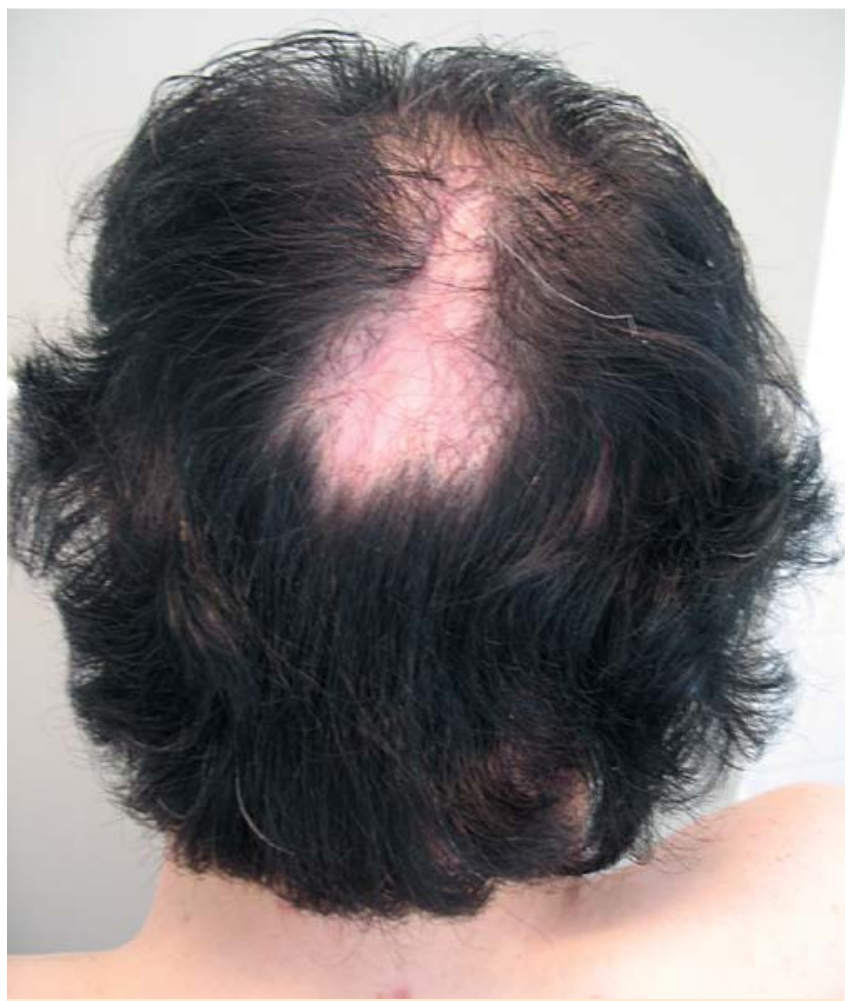

Abb. 3 Ulzeröses PG am Capillitium. Atrophische Narbe 10 Monate nach systemischer Therapie mit Solu Decortin H, Azathioprin und Mesalazin.

Vorhandensein assoziierter Erkrankungen und dem Ausschluss anderer ulzerativer Prozesse, die ein ähnliches Erscheinungsbild zeigen.

Typische auszuschließende Differenzialdiagnosen sind beispielsweise Vaskulitis, Infektionen, ischämische oder venöse Ulzera etc. [4]. Aufgrund des typischen klinischen Erscheinungsbildes, der erstmalig aufgetretenen Symptomatik einer CU und der atypischen Lokalisation am Capillitium gehen wir in diesem Fall von einer ulzerösen Variante aus.

Die ulzeröse bzw. klassische Form präsentiert sich mit schmerzhaften, großflächigen Ulzera mit eitriger Basis und unterminiertem lividen Rand. Initiale HV wie entzündliche Pusteln oder Knoten zerfallen rasch nekrotisch und es bilden sich charakteristische Ulzera mit unterminiertem, bläulich-violettem, meist druckschmerzhaften Randwall, der häufig von einem Erythem und Ödem umgeben wird [4]. Am häufigsten sind die unteren Extremitäten und der Körperstamm betroffen [4,6], in seltenen Fällen können auch atypische Lokalisationen wie der Kopf und Nacken [7,14] sowie die Genitale [5] betroffen sein.

Die Histologie der frühen Stadien zeigt eine perivaskuläre und intramurale Lymphozyteninfiltration mit Neutrophilen und fibrinoider Nekrose. Fortgeschrittene Stadien sind charakterisiert durch Abszessformationen und „epithelial undermining“ [6]. Mykologische und bakterielle Untersuchungen der Ulzera lassen keine ursächlichen Erreger erkennen. Die Histologie ist stets unspezifisch. Das PG ist eine klinische Diagnose.

Seltene Fälle mit PG der Kopfhaut wurden erstmalig 1948 [13], 1974 [7] und zuletzt 2005 [14] beschrieben. Ricketts weist schon 1948 darauf hin, dass eine adäquate Therapie der assoziierten Erkrankung obligat ist und es nur so zu einer langfristigen Besserung der HV mit rezidivfreiem Verlauf kommen kann. Er beschreibt einen Fall, bei dem sich ebenfalls kurze Zeit nach Erstdiagnose einer CU multiple rötliche Knoten und Pusteln am Nacken, Stirn, Brust und Rücken bildeten. Die Therapie mit strenger Diät kombiniert mit Antibiotikagabe blieb erfolglos. Die Anlage eines Ileostomas führte nur kurzfristig zur Besserung und es traten zirka ein Jahr später bei Wiederaufflammen der CU auch wieder ähnliche HV auf. Allein eine Kolektomie brachte langfristig Besserung [13]. Auch in unserem Fall führte erst eine systemische Glukokortikoidtherapie begleitet von einer Mesalazingabe speziell zur Behandlung der CU und eine spätere Azathioprinmedikation zur kompletten Remission. Zusammenfassend ist darauf hinzuweisen, dass sowohl Art als auch Schweregrad der assoziierten Erkrankung für das PG von prognostischer Bedeutung sind. In vielen Fällen führt die erfolgreiche Therapie der assoziierten Erkrankung zu einer Verbesserung oder kompletten Abheilung des PG [11].

\section{Abstract}

\section{Pyoderma gangrenosum of the Scalp in a Patient with Ulcerative Colitis $\nabla$}

We present a 42-year-old male patient with pustules at the backside of the scalp, which occurred during a gastroenterological inpatient treatment for clarification of repeated bleeding diarrhoeas and culminated to painful ulcerations on the scalp. Pyoderma gangrenosum was diagnosed. Further detailed diagnostic identified ulcerative colitis (CU) as the underlying disease. Oral treatment with steroids and Mesalazin for treatment of ulcerative colitis followed by an oral azathioprine-medication induced complete recovery. 


\section{Literatur}

1 Wollina $U$. Pyoderma gangrenosum - a review. Orphanet J Rare Dis 2007; $15: 19-27$

2 Wollina $U$. Clinical management of pyoderma gangrenosum. Am J Clin Dermatol 2002; 3: 149-158

3 Hickman JG. Pyoderma gangrenosum. Clin Dermatol 1983; 1: 102-113

4 Conrad C, Trüeb RM. Pyoderma gangrenosum. J Dtsch Dermatol Ges 2005; 3: $334-342$

5 Sanusi ID, Gonzalez E, Venable DD. Pyoderma gangrenosum of penile and scrotal skin. J Urol 1982; 127: 547-549

6 Crowson AN, Mihm Jr MC, Magro C. Pyoderma gangrenosum: a review. J Cutan Pathol 2003; 30: 97-107

7 Peachey RD. Proceedings: Pyoderma gangrenosum of scalp. Br J Dermatol 1974; 90: 106-108

8 Powell FC, Su WP, Perry HO. Pyoderma gangrenosum: classification and management. J Am Acad Dermatol 1996; 34: 395-409 ; quiz 410-412
9 Powell FC, Perry HO. Pyoderma gangrenosum in childhood. Arch Dermatol 1984; 120: $757-761$

10 Brunsting LA, Goeckermann WA, O'Leary PA. Pyoderma (ecthyma) gangrenosum: clinical and experimental observations in 5 cases occurring in adults. Arch Derm Syph 1930; 22: 655-680

11 Hasselmann DO, Bens G, Tilgen W, Reichrath J. Pyoderma gangrenosum: clinical presentation and outcome in 18 cases and review of the literature. J Dtsch Dermatol Ges 2007; 5: 560-564

12 Mlika RB, Riahi I, Fenniche $S$ et al. Pyoderma gangrenosum: a report of 21 cases. Int J Dermatol 2002; 41: 65-68

13 Ricketts WE, Kirsner JB, Rothman S. Pyoderma gangrenosum in chronic non-specific ulcerative colitis; a report of three cases. Am J Med 1948; 5: $69-75$

14 Poenitz N, Tadler D, Klemke C-D et al. Ulceration of the scalp: a unique manifestation of pyoderma gangrenosum. J Dtsch Dermatol Ges 2005: 3: $113-116$ 